\title{
New frontiers in environmental noise research
}

DOI 10.2478/noise-2014-0001

Environmental noise has been an issue since ancient times. The first documented noise ordinance was made by the council of the Greek province of Sybaris in the 6th century BC, banning, among other things, roosters. One of the earliest attempts to define noise regulation policy in the modern era dates back to 1929, when the Noise Abatement Commission was established in New York.

Noise impact on the world population is increasing. This is, in large part, due to the growing spread of anthropogenic influences including particularly factories and mechanical transportation. Urbanization remains a dynamic process, especially because of the rapid growth of developing countries: the United Nations estimates that more than two thirds of the inhabitants of the world will live in urban areas by 2050. Furthermore, the European Commission has recently assessed that only $10 \%$ of the world's land area can be reached in more than 48 hours from a city with a population $>50,000$. This is facilitated by the continuous development of a widespread and interconnected transportation network. According to the World Bank, the number of flights has increased by one third in the last decade.

Increasing urbanization can be associated with a greater variety of noise and some negative health issues. In fact, the World Health Organization claims that traffic noise annoys one in three Europeans during the course of a given day. One in five will have their sleep disturbed for the same reason. The United States Environmental Protection Agency claimed that 30 million Americans are exposed to non-occupational noise high enough to cause hearing loss and 44 million Americans live in homes impacted by aircraft or highway noise. Continued noise exposure has been linked to cardiovascular diseases, cognitive impairment in children, sleep disturbance, and tinnitus.

To counteract the detrimental effects of noise, several regulatory policies have been defined at local, national and supranational level worldwide. In 2002, the European

Francesco Asdrubali: Editor, Noise Mapping

Department of Engineering, University of Perugia, Italy
Union issued the Directive 2002/49/EC for the evaluation and regulation of environmental noise through common mapping activities and action plans. In the USA, the wellknown Noise Control Act was enacted by Congress in 1972 and amended in 1978; later, the primary responsibility for regulating noise was delegated to state and local governments.

The standard approach for noise regulation is to set maximum thresholds for SPL and to require mitigation activities when the limits are surpassed. Noise source characterization and modeling alongside noise propagation and mapping are essential activities to design effective noise mitigation measures. However, silence cannot be the goal of these measures, since sounds characterize a place in meaningful ways, influencing how it is experienced. The study of soundscape, that is the acoustic environment as perceived, experienced and/or understood by people in context, is in many respects dedicated to developing measures that improve the quality of life. As such, soundscape research is complementary to (and in some cases, a key component of) environmental noise strategies.

Within this context, I am honored to introduce the new journal Noise Mapping, which aims to become the main publishing option for authors writing on noise mapping and related topics. We hope this journal will grow into a hub integrating the relevant research communities in the fields of environmental noise and soundscape studies. A LinkedIn ${ }^{\circledR}$ group named "Noise Mapping" has been created to foster and encourage discussion and debate on such topics, connecting researchers, policy advisors, and scientists around common themes.

This journal aims to promote and disseminate knowledge on noise mapping through the publication of high quality peer-reviewed papers focusing on all the various aspects of environmental acoustics, and in particular:

- noise mapping and noise action plans: case studies;

- models and algorithms for source characterization and outdoor sound propagation: proposals, applications, comparisons, round robin tests;

- local, national and international policies and good practices for noise mapping, planning, management and control;

- evaluation of noise mitigation actions;

- evaluation of environmental noise exposure; 
- actions and communications to increase public awareness of environmental noise issues;

- outdoor soundscape studies and mapping;

- classification, evaluation and preservation of quiet areas;

- characterization of the emissions of the following sources: roads, railways, airports and aircrafts, harbors and ships, industrial plants, leisure and night-life activities, urban areas, natural sound sources.

The Members of the Noise Mapping Editorial Advisory Board are recognized leading scientists, covering different geographic areas and research fields of environmental acoustics. I would like to thank all of them for their valuable contributions, especially in this initial phase.

Noise Mapping is open-access and offers a fast and comprehensive peer-review. To ensure that the journal has the largest possible impact in this early phase, no publication fees will apply until the end of 2015. Special issues on emerging topics are also going to be a part of this exciting endeavor.

I am looking forward to receiving your papers! 